FORMATION Formation emploi

Revue française de sciences sociales

125 | Janvier-Mars 2014

Pêle-Mêle

\title{
Le rôle de l'accompagnement dans la réussite des parcours de validation des acquis de l'expérience
}

The role of the individual accompaniment in the success of the Validation of Experential Learning

Die Rolle der Betreuung bei einem erfolgreichen Durchlauf zur qualifizierenden Anerkennung erworbener Kompetenzen

El papel del acompañamiento en el éxito de los itinerarios de validación de la experiencia adquirida

\section{Nathalie Havet}

\section{CpenEdition} Journals

Édition électronique

URL : http://journals.openedition.org/formationemploi/4147

DOI : 10.4000/formationemploi.4147

ISSN : 2107-0946

Éditeur

La Documentation française

Édition imprimée

Date de publication : 25 mars 2014

Pagination : 47-68

ISSN : 0759-6340

Référence électronique

Nathalie Havet, «Le rôle de l'accompagnement dans la réussite des parcours de validation des acquis de l'expérience », Formation emploi [En ligne], 125 | Janvier-Mars 2014, mis en ligne le 31 mars 2016, consulté le 30 octobre 2020. URL : http://journals.openedition.org/formationemploi/4147 ; DOI : https://doi.org/10.4000/formationemploi.4147 


\title{
Le rôle de l'accompagnement dans la réussite des parcours de validation des acquis de l'expérience
}

\author{
Nathalie Havet \\ Maître de conférences en sciences économiques à l'université Lumière Lyon 2 et membre du \\ Groupe d'Analyse et de Théorie Economique (GATE) Lyon Saint-Etienne (UMR CNRS 5824)
}

Résumé

Le rôle de l'accompagnement dans la réussite des parcours de validation des acquis de l'expérience

Notre article cherche à décrire les parcours des candidats à la validation des acquis de l'expérience (VAE) et le rôle joué par les entretiens conseil et les prestations d'accompagnement sur chacune des étapes de ce dispositif. Pour ce faire, les données sur l'ensemble des individus ayant déposé un dossier de candidature à la VAE auprès des principaux valideurs de la région Rhône-Alpes, entre 2007 et 2009, ont été mobilisées. Il ressort que la VAE remplit son rôle de qualification des personnes peu diplômées. L'accompagnement durant la procédure semble un atout incontestable dans la réussite de ces parcours de VAE.

Mots clés : validation des acquis, accompagnement professionnel, Rhône-Alpes

Abstract

The role of the individual accompaniment in the success of the Validation of Experential Learning

Our paper empirically investigates the different steps of the Validation of Experential Learning (VAE) procedure. The analysis is based upon the exhaustive data of the candidates for VAE in 2007-2009 in Rhone-Alps Region. Our results show that VAE plays a true role for the unskilled workers. The individual accompaniment during the validation is decisive for the success of the procedure.

Keywords: validation of acquired skills, coaching, Rhône- Alpes

Journal of Economic Literature: I 28

Traduction : Auteur 
La loi de Modernisation Sociale du 17 janvier 2002 a institué, en France, le droit à la validation des acquis de l'expérience (VAE). Il permet d'obtenir tout ou partie d'un diplôme ou d'une certification sans devoir suivre de cursus de formation initiale ou de formation professionnelle continue. Avec ce dispositif, le législateur a créé les conditions d'un accès individuel à la formation tout au long de la vie. Sa volonté a été de mettre la progression des salariés au cœur du système de formation en favorisant l'articulation entre formation, certification professionnelle et déroulement des carrières. Un des principaux objectifs affiché était de favoriser le signalement des compétences individuelles innées et acquises via l'ensemble des expériences sur le marché du travail et/ou dans le milieu social ${ }^{1}$. Les entreprises, en identifiant plus aisément les compétences des candidats dans un emploi, peuvent ainsi mieux les différencier des autres postulants (effet signal) et leur permettre une évolution de carrière, quand le diplôme n'est pas tout simplement la condition sine qua non de leur progression dans les grilles salariales de la branche. Pour les personnes pas ou peu qualifiées, souvent en difficulté d'insertion sociale et professionnelle, la VAE peut constituer un outil précieux dans la sécurisation de leurs parcours. Par exemple, les salariés « faisant fonction ", c'est-à-dire qui exercent souvent avec efficacité les mêmes activités que les personnes diplômées peuvent, grâce à la VAE, acquérir en cours de carrière le diplôme nécessaire à leur évolution statutaire et à leur progression salariale. Mais, plus généralement, quel que soit le niveau de qualification, la VAE peut constituer un levier dans la gestion du déroulement de carrière (Ballet, 2009 ; Labruyère et al., 2002) ; les candidats peuvent en effet en attendre des retombées en termes de reconnaissance sociale, de mobilité professionnelle, à l'intérieur ou à l'extérieur de l'entreprise, ou une réorientation professionnelle.

En France, entre 2002 et 2011, près de 230000 candidats ont été certifiés par la voie de la VAE (Legrand, 2012). Après une forte progression entre 2003 et 2005, le nombre de candidats certifiés a oscillé, depuis 2007, entre 28000 et 32 000, loin cependant de l'objectif gouvernemental de 60000 certifications par an (Besson, 2008). Même si les premiers niveaux de qualification reconnus sont majoritairement plébiscités - près d'un candidat sur deux vise un diplôme de niveau V (CAP, BEP - certificat d'aptitude professionnelle, brevet d'études professionnelles - ou équivalent) -, les validations de l'enseignement supérieur ont fortement progressé depuis la mise en place de la VAE : de 1040 en 2002, elles sont passées à plus de 4000 en 2011 (Le Roux, 2012).

Bien que la VAE semble avoir atteint un certain rythme de croisière, les premiers bilans ont pointé des difficultés inhérentes à ce dispositif (Kogut-Kubiak et al., 2006 ; Besson, 2008). Parmi elles, la montée en puissance du nombre de certifications au titre de la VAE semble se heurter non seulement au nombre limité de dossiers déposés, sans doute lié à un manque d'informations préalables au processus, mais aussi à un phénomène

1. L'intérêt de ce dispositif peut se lire à la lumière des arguments de la théorie du signal (Spence, 2002 ; Giret et Lemistre, 2008). 
de sélection opérée une fois la candidature réalisée. En particulier, on observe de nombreuses déperditions de candidats tout au long de la procédure, jugée longue et complexe. Dès lors, l'étude des différentes étapes du dispositif de VAE et la compréhension du processus de sélection et des réels bénéficiaires semblent primordiales.

Cet article s'inscrit dans la continuité des études des profils de candidats à la VAE réalisées par Labruyère (2006a), Bonaïti (2008) et Havet (2014). Il permet d'actualiser les conclusions de Labruyère (2006a), qui analysait les prémisses du système de VAE à partir de données de 2003-2004. Contrairement à Bonaïti (2008) qui examinait le parcours des candidats visant un titre de niveau $V$, notre étude adopte une vision plus globale du processus en ne se limitant pas à un niveau de diplôme spécifique. Elle s'appuie sur la même base de données rhônalpines que Havet (2014); cependant, elle la complète en centrant l'analyse sur les rôles potentiels des entretiens conseils et de l'accompagnement aux différentes phases du processus. Il s'agit de déterminer si, pour les pouvoirs publics, il est pertinent de les renforcer et de les utiliser comme moyens d'action pour améliorer l'efficacité de la VAE.

La première partie décrit le dispositif de validation des acquis de l'expérience. Les deuxième et troisième parties utilisent les données administratives 2007-2009 de la région Rhône-Alpes, collectées en 2011, pour dresser un bilan quantitatif global et par diplôme de la VAE. Enfin, dans la dernière partie, nous tentons de déterminer si les entretiens conseils et l'accompagnement constituent de réels atouts à toutes les étapes de la procédure de VAE.

\section{La VAE : de l'information initiale au passage devant le jury}

La Validation des Acquis de l'Expérience (VAE), prévue par le Code du travail et le Code de l'éducation, est un droit individuel, pour toute personne engagée dans la vie active, à demander, sur la base de son expérience professionnelle ou de ses activités, l'obtention de tout ou partie d'un diplôme ou d'une certification à finalité professionnelle figurant sur la liste du Répertoire national des certifications professionnelles (RNCP). Peuvent être prises en compte, au titre de la validation, l'ensemble des compétences professionnelles acquises dans l'exercice d'une activité en rapport direct avec le contenu du diplôme ou du titre, que cette activité ait été salariée, non-salariée ou bénévole. La condition pour pouvoir entamer une démarche de VAE est de justifier de trois années d'expérience (continue ou discontinue, à temps plein ou à temps partiel) en lien avec la certification visée.

La VAE recouvre plusieurs étapes essentielles, qui vont de l'information initiale du futur candidat à l'examen de son dossier par un jury, en passant par la constitution de ce dossier. Plus précisément, en amont de l'action de validation proprement dite, la 
première démarche d'un candidat potentiel est de s'informer sur la VAE et de définir son projet professionnel. Il peut trouver un premier niveau d'information dans les différentes structures d'information et d'orientation régionales (Pôle Emploi, Missions locales, chambres consulaires, Centre d'Information et d'Orientation, Maisons de l'Information sur la Formation et l'Emploi, Organismes Paritaires Collecteurs Agréés, etc).

En région Rhône-Alpes, le service d'Information-Conseil sur la VAE s'est structuré, depuis 2007, autour d'une part, des Points Relais d'Information Conseil VAE (PRIC) qui sont labellisés et assurent une information approfondie et un conseil individualisé ; d'autre part, des Points Relais d'Information VAE (PRI) qui ont vocation à assurer une information plus générale des publics. En particulier, le candidat peut obtenir gratuitement, au sein des PRIC, un conseil personnalisé pour déterminer si le projet d'accès à une certification par la voie de la VAE est pertinent au regard de ses objectifs; il s'agit de faire le point sur son parcours professionnel et ses attentes.

Au cours de cet entretien, le conseiller peut notamment éclairer le candidat sur le choix des certifications correspondant à son expérience. Les activités qu'il a exercées doivent en effet correspondre, en tout ou en partie, aux activités décrites dans le "référentiel d'activités » de la certification visée. Or, l'identification de la certification appropriée est souvent entravée par l'existence de plus de 4800 diplômes dans le RNCP. Par ailleurs, les personnes au parcours professionnel chaotique, dont les compétences renvoient souvent à des blocs de compétences de certification différentes, peuvent peiner à identifier la certification adéquate. Pourtant, cette étape est déterminante pour la suite du processus : des candidats qui s'orientent trop précocement vers un certificateur sans avoir consulté un conseiller se retrouvent enfermés dans leur démarche de VAE en raison d'une erreur dans le choix initial de la certification (Besson, 2008).

Si, à l'issue de la phase d'information, le candidat décide de s'engager dans la démarche de VAE, il doit absolument choisir la certification qu'il vise. Une fois le valideur identifié (organisme qui organise la session et effectue l'évaluation), il doit le contacter afin de lui faire part de sa candidature. C'est à partir de cette étape que commence la VAE proprement dite.

Les candidats à la VAE doivent déposer, auprès du certificateur concerné, un premier dossier de candidature dit "dossier de recevabilité ", qui retrace leur parcours professionnel. Il est en général demandé d'apporter des preuves des activités exercées en joignant tous les justificatifs officiels (bulletins de salaires, attestations d'employeur ou d'association, etc.) attestant de la durée de chaque activité et de son contenu. A partir de ces éléments, le valideur se prononce sur la recevabilité de la demande de VAE : le dossier est déclaré recevable si le candidat remplit les conditions réglementaires d'accès à un parcours de VAE. 
En particulier, le certificateur vérifie deux points : la durée de l'expérience du candidat et l'adéquation entre les activités exercées et celles décrites dans le référentiel de la certification. La décision de recevabilité ne préjuge en aucun cas du résultat de la validation, prononcé par le jury. Si la demande du candidat est jugé recevable, la procédure de VAE se poursuit : le certificateur remet au candidat le " dossier de présentation de l'expérience " qui décrit de façon détaillée les activités et qui sera soumis au jury de la certification.

L'objectif de ce second dossier est de permettre au jury d'apprécier si les connaissances, les compétences et les aptitudes acquises par l'expérience sont comparables aux compétences attendues d'un titulaire de la certification visée. Une fois le dossier constitué et déposé, il existe différentes modalités de mise en œuvre de la VAE selon l'organisme certificateur et le titre visé : examen du dossier et entretien avec le jury, et parfois mise en situation professionnelle du candidat. Lorsqu'il y a une mise en situation, les compétences du candidat sont évaluées en situation de travail réelle ou reconstituée. Au vu de ces éléments, le jury peut décider d'une validation totale, d'une validation partielle ou refuser la validation.

Par validation totale, le jury reconnaît que le candidat a acquis, grâce à son expérience, les compétences attendues des détenteurs de la certification visée et le déclare donc titulaire du diplôme. En cas de validation partielle des acquis, des prescriptions (formation ou travail complémentaire) sont proposées au candidat en vue d'obtenir la totalité du diplôme dans un délai de cinq ans. Le refus de validation signifie quant à lui que les acquis du candidat sont insuffisants ou ne correspondent pas à la certification visée.

Pour élaborer ce second dossier et préparer l'entretien devant le jury, une prestation d'accompagnement peut être proposée. Elle est assurée, soit par les certificateurs euxmêmes, soit par des prestataires publics ou privés. Elle peut prendre différentes formes (entretiens individuels, réunions collectives ou un mixte des deux), avec une qualité et une intensité variables (Personnaz, Quintero et Séchaud, 2005). L'accompagnement est le plus souvent facultatif et payant. Il ne concerne en général pas l'amont de la procédure, c'est-à-dire la constitution du dossier de recevabilité.

Nos données pour la région Rhône-Alpes, extraites de la base Résovalideur (encadré 1) pour la période 2007-2009, nous permettent de suivre les candidats à la VAE à partir du dépôt de dossier de recevabilité. Par conséquent, sont renseignés la décision de recevabilité, le fait d'être passé ou non devant le jury sur la période d'observation et si oui, la décision du jury. 


\section{Encadré 1 : La base Résovalideur de la Région Rhône-Alpes}

Même si un effort d'harmonisation dans la collecte des données relatives à la VAE est en train d'être réalisé entre les différents acteurs, l'information disponible reste parcellaire. Les données mobilisées sont extraites de la base Résovalideur, construite à partir des dossiers individuels déposés auprès des principaux valideurs de la région Rhône-Alpes, région en pointe dans la collecte et I'harmonisation des données de VAE.

Pour la population totale ou active, la région Rhône-Alpes représente 10 \% du national et environ $9 \%$ du total national pour les démarches de VAE (Becque, 2010). Les organismes valideurs participant à ce projet sont au nombre de 12 : le ministère de l'Emploi, les dispositifs académiques de validation des acquis (DAVA) de Lyon et de Grenoble, les universités Lyon 1, Lyon 2, Lyon 3, Grenoble 2, Grenoble 3, Saint-Etienne et de Savoie, la direction régionale de l'Agriculture, de I'Alimentation et de la Forêt (DRAFF) et la direction régionale de la Jeunesse, des Sports et de la Cohésion sociale (DRJSCS). L'université de Grenoble 1 a dû renoncer à son implication dans le projet de suivi des parcours de VAE pour des raisons liées à son système informatique. Les données fusionnées nous ont été fournies par le Pôle Rhône-Alpes de l'Orientation (PRAO).

Notre échantillon est constitué de l'ensemble des candidats résidant en région Rhône-Alpes qui ont établi un dossier et ont obtenu une décision de recevabilité (positive ou négative) entre le $1^{\text {er }}$ janvier 2007 et le 31 décembre 2009 pour l'un des valideurs participant au Résovalideur, soit 18941 individus. Les candidats originaires d'une autre région et ayant validé une certification en Rhône-Alpes ne sont pas pris en compte. Les données exploitées sont celles qui étaient disponibles dans Résovalideur à la date d'exportation du 14 avril 2011. Ainsi certains candidats n'ont pas forcément achevé leur démarche VAE.

Ces données fournissent des informations longitudinales sur les éléments de biographie des individus, permettant de reconstituer leur parcours de VAE, depuis la décision de recevabilité jusqu'à la décision du jury de validation. Elles comportent aussi des informations démographiques, telles que le sexe, l'âge, le statut professionnel (demandeur d'emploi, salarié, non-salarié) ou encore le niveau de diplôme visé par la démarche de VAE. Le niveau de diplôme initial des candidats ne peut en revanche pas être exploité car il est très peu renseigné par les valideurs. De même, il n'est pas possible de déterminer si un candidat a réellement abandonné sa démarche de VAE car les valideurs n'ont pas tous les mêmes délais de référence pour conclure à l'abandon.

\section{Entretien conseil et accompagnement}

Chaque fiche administrative des candidats a été enrichie de données provenant des Points Relais d'Information Conseil (PRIC). Le rapprochement entre les données des valideurs et celles des PRIC s'est effectué via la création d'une clé cryptée (nom-prénom-date de naissance-code postal). II a essentiellement permis de savoir si les candidats ayant déposé un dossier avaitent pu bénéficier d'un entretien conseil au sein d'un PRIC.

L'information disponible concernant l'accompagnement après la décision de recevabilité n'est renseignée que partiellement. Certains valideurs, comme les Affaires sanitaires et sociales, délèguent complètement leur prestation d'accompagnement à des structures indépendantes et ne peuvent donc fournir de données sur cette prestation.

C'est pourquoi notre analyse de l'impact des entretiens conseils et de l'accompagnement n'a été effectuée qu'à partir des données issues de l'Education nationale (Enseignement secondaire : DAVA de Lyon et Grenoble), de Jeunesse et Sports (DRJSCS) et du ministère de l'Emploi. Elle est donc limitée. L'échantillon est ainsi réduit à 10930 candidats. Notons que nos résultats peuvent sous-estimer le rôle de l'accompagnement, puisque le valideur n'a pas forcément eu connaissance des prestations d'accompagnement effectuées par les organismes privés. En outre, nous n'avons aucune information sur le contenu, la qualité et l'intensité de cet accompagnement. 


\section{Une déperdition des candidats après l'étape de recevabilité}

Parmi les 18941 candidats à la VAE recensés dans la base Résovalideur, $90 \%$ ont un dossier qui est jugé répondre aux critères de recevabilité, c'est-à-dire trois années d'expérience en lien avec la certification. Ce taux, relativement élevé, peut en partie s'expliquer par le fait que certains valideurs, notamment de l'enseignement secondaire, examinent la faisabilité du projet du candidat avant que celui-ci ne dépose son dossier de recevabilité. Ainsi, si l'expérience du candidat ne correspond pas suffisamment au référentiel de la certification visée, le candidat a la possibilité de retravailler son projet avant de déposer son dossier de recevabilité ou bien d'abandonner sans que cela soit comptabilisé parmi les recevabilités négatives.

En outre, d'autres valideurs, comme l'Education nationale ou le ministère de l'Emploi, gèrent cette étape de recevabilité de façon plus administrative : ils se contentent de vérifier si le candidat peut justifier des trois années d'expérience, sans examiner l'adéquation de ses compétences avec la certification visée - cette adéquation sera examinée par le jury. Nous trouvons ainsi des taux de recevabilité positive particulièrement élevés $(97 \%)$ pour les candidats visant un titre du ministère de l'Emploi et, dans une moindre mesure, pour ceux candidatant auprès de l'Education nationale et de l'Enseignement secondaire (93\%). De même, le faible taux de recevabilité positive des candidats dans le secteur Jeunesse et Sports (77 \%) s'explique par le traitement différencié de la recevabilité chez ces valideurs. Par exemple, la direction régionale de la Jeunesse, des Sports et de la Cohésion sociale (DRJSCS) saisit les informations du candidat et le considère comme non recevable tant qu'elle n'a pas toutes les pièces justificatives. Cette procédure maintient temporairement une part relativement importante de candidats dans cette situation de non-recevabilité, sans hypothéquer leurs chances de passer cette étape : une fois fournies les pièces manquantes, le dossier du candidat pourra être considéré comme recevable (PRAO, 2011).

Malgré une faible proportion de candidats éliminés du processus de VAE à l'étape de recevabilité, relativement peu de candidats se sont présentés en jury et ont obtenu une décision sur leur validation d'acquis d'expérience. Sur les 17022 candidats recevables entre 2007 et 2009, seulement 47 \% (8 026 candidats) avaient été présentés en jury à la fin des trois années ${ }^{2}$. Les raisons de ce phénomène sont multiples.

Premièrement, à la suite d'une recevabilité positive, les candidats doivent constituer leur " dossier de présentation de l'expérience » et un jury doit être organisé, ce qui prend un certain temps. Ainsi, certains candidats ayant été jugés recevables, notamment le plus récemment, sont encore en cours de rédaction de leur dossier ou sont en attente de la date

2. Ce taux de passage en jury varie néanmoins de $33 \%$ pour les Affaires sanitaires et sociales à $58 \%$ pour l'Education nationale/Enseignement secondaire. 
de jury. Pour les candidats présentés en jury ${ }^{3}$, le délai moyen entre la recevabilité positive et le passage en jury était en effet de l'ordre d'une année. Mais cette moyenne cache des disparités : $52 \%$ des candidats à la VAE ont mis entre un à deux ans à passer devant un jury et $5 \%$ plus de 24 mois.

Deuxièmement, de nombreux candidats recevables abandonnent leur projet de VAE après l'étape de recevabilité, notamment du fait d'évènements familiaux, des délais jugés trop longs, d'un changement de situation professionnelle ou d'orientation, ou simplement à cause du travail de réflexion et de rédaction exigé pour la constitution du dossier (Personnaz, Quintero et Séchaud, 2005). Malheureusement, la base de données ne permet pas d'identifier ceux qui ont abandonné de ceux dont la démarche VAE est encore en cours.

Parmi les candidats présentés en jury, $61 \%$ (4 851 candidats) ont validé totalement la certification visée à la fin des trois années, $28,5 \%$ (2 246) partiellement et 10,5\% (829) ont obtenu un refus. Le taux de validation complète pour la région RhôneAlpes est très proche de celui du niveau national, qui s'élevait à $58 \%$ en 2010 et 2011 (Legrand, 2012). Les valideurs qui avaient les taux de recevabilité positive les plus élevés se démarquent aussi par leur taux élevé de validation totale : 80,5\% pour le ministère de l'Emploi et $67 \%$ pour l'Enseignement secondaire. Nous observons ainsi une déperdition importante de candidats tout au long du processus de VAE. Sur les presque 20000 candidats ayant entamé une démarche de VAE entre 2007 et 2009 en Rhône-Alpes, seulement 26 \% se sont vus délivrer une validation totale. D’ailleurs, Besson (2008) notait que nationalement, il y avait comparativement davantage de déperditions parmi les candidats à la VAE que parmi les stagiaires de la formation continue, selon les données disponibles pour les diplômes de l'Education nationale.

\section{La VAE \\ 3 au service d'un public peu qualifié}

Le processus de VAE semble remplir son premier objectif d'attirer des adultes peu qualifiés. Près d'un candidat sur deux (48\%) à la VAE vise un diplôme de niveau V et seulement $3 \%$ visent un diplôme équivalent à une licence ou un master (niveau I ou II). Le diplôme le plus prisé est le diplôme d'Etat d'aide soignant; viennent ensuite le diplôme d'Etat d'auxiliaire de vie sociale, le CAP petite enfance, le diplôme d'Etat d'auxiliaire de puériculture et les diplômes d'Etat d'éducateur spécialisé et d'éducateur de jeunes enfants (tableau 1). Ces six certifications représentent à elles seules $45 \%$ de l'ensemble des certifications demandées. Comme parmi ces certifications, les quatre

3. Sur les 7926 candidats, seulement 7754 délais ont pu être calculés du fait d'informations manquantes. 
premières sont de niveau $\mathrm{V}$ - les diplômes d'Educateur sont de niveau III -, il n'est pas surprenant que ce niveau de diplôme visé soit largement majoritaire. Néanmoins, parmi les 15 certifications les plus demandées en VAE se classent trois diplômes de niveau bac + 2: le BTS (brevet de technicien supérieur) Assistant de gestion PME-PMI, le BTS Aide de direction et le BTS Comptabilité gestion des organisations.

Les demandes de VAE semblent avoir pour objectif une valorisation directe sur le marché du travail. Pour la majorité des demandes, il s'agit de diplômes appartenant aux secteurs du travail social, des services à la personne, du tertiaire administratif ou de la coiffure, des secteurs relativement en tension, avec de nombreux postes à pourvoir. Ce sont aussi des secteurs très féminisés, ce qui explique la prédominance des femmes (75\%) parmi les candidats à la VAE.

Nombre de certifications visées sont très recherchées car elles sont réglementairement obligatoires à l'exercice du métier. C'est par exemple le cas du BP Coiffure pour ouvrir son salon ou du BP de la Jeunesse, de l'Education populaire et du Sport (BP JEPS)/ Loisirs tous publics, pour exercer en tant qu'animateur. La sécurisation de leurs parcours professionnels, en particulier via un accès à la titularisation dans l'emploi public, est certainement un objectif poursuivi par un certain nombre de candidats au diplôme d'Educateur spécialisé (de Rozario, 2013) ou au CAP Petite enfance. La détention de ce dernier permet notamment d'accéder par concours aux emplois d'agents territoriaux spécialisés des écoles maternelles (ATSEM). Pour répondre à des besoins de main-d'œuvre importants, les établissements sociaux et médico-sociaux ont souvent recours à des personnels qui peuvent être amenés à assumer certaines tâches dévolues en principe aux détenteurs de ces diplômes (aide-soignant, éducateur spécialisé, etc.). Il n'est donc pas surprenant de les retrouver parmi les certifications les plus visées. La VAE pourrait donc jouer son rôle de reconnaissance de l'expérience de ces salariés "faisant fonction».

Toutefois, les diplômes les plus demandés ne sont pas les diplômes les plus validés. Parmi les 15 certifications les plus demandées, le titre d'Assistant de vie aux familles présente le taux de validation totale le plus élevé (87\%). Cette certification est souvent visée dans le cadre de projets collectifs de VAE, qui tendent à accroître la motivation et la ténacité des candidats et donc l'obtention de meilleurs résultats de validation par rapport aux procédures de VAE individuelles. En effet, dans ces projets collectifs à l'initiative de l'employeur, il est proposé aux personnes, même si la rédaction des dossiers reste individuelle, de bénéficier d'une logistique mobilisée pour l'ensemble des salariés ; les acteurs se concertent pour définir les calendriers et optimiser les différentes séquences de la VAE (Beaupère et Podevin, 2012). Viennent ensuite le BTS Assistant de gestion PME-PMI (74 \%), le bac pro Secrétariat (73\%), le BP JEPS, le BTS Comptabilité gestion des organisations (66\%) et le CAP Petite enfance (62\%). 
Tableau 1 : Les 15 certifications les plus demandées par les candidats à la VAE

\begin{tabular}{|c|c|c|c|c|c|c|c|c|}
\hline $\begin{array}{c}\text { Certifications } \\
\text { visées }\end{array}$ & $\begin{array}{l}\text { Dossiers de } \\
\text { recevabilité }\end{array}$ & $\begin{array}{c}\text { Recevabilité } \\
\text { positive }\end{array}$ & $\begin{array}{c}\text { Décisions } \\
\text { de jury }\end{array}$ & $\begin{array}{l}\text { Val. } \\
\text { Totale }\end{array}$ & $\begin{array}{c}\text { Val. } \\
\text { Partielle }\end{array}$ & Refus & $\begin{array}{l}\text { Entretiens } \\
\text { conseil } \\
\text { PRIC }\end{array}$ & $\begin{array}{l}\text { Accompa- } \\
\text { gnement }\end{array}$ \\
\hline $\begin{array}{l}\text { DE Aide } \\
\text { Soignant }\end{array}$ & 2285 & $86 \%$ & 571 & $32 \%$ & $57 \%$ & $11 \%$ & $10 \%$ & $\mathrm{Nd}$ \\
\hline $\begin{array}{l}\text { DE Auxiliaire } \\
\text { de vie sociale }\end{array}$ & 1906 & $93 \%$ & 794 & $60 \%$ & $36 \%$ & $4 \%$ & $8 \%$ & $\mathrm{Nd}$ \\
\hline $\begin{array}{l}\text { CAP Petite } \\
\text { Enfance }\end{array}$ & 1469 & $99 \%$ & 923 & $62 \%$ & $36 \%$ & $4 \%$ & $19 \%$ & $54 \%$ \\
\hline $\begin{array}{l}\text { DE } \\
\text { Auxiliaire de } \\
\text { puériculture }\end{array}$ & 1140 & $76 \%$ & 293 & $27 \%$ & $62 \%$ & $11 \%$ & $11 \%$ & $\mathrm{Nd}$ \\
\hline $\begin{array}{l}\text { DE Educateur } \\
\text { Spécialisé }\end{array}$ & 1090 & $84 \%$ & 475 & $59 \%$ & $28 \%$ & $12 \%$ & $9 \%$ & $23 \%$ \\
\hline $\begin{array}{l}\text { DE Educateur } \\
\text { de Jeunes } \\
\text { Enfants }\end{array}$ & 648 & $77 \%$ & 112 & $28 \%$ & $43 \%$ & $29 \%$ & $9 \%$ & $\mathrm{Nd}$ \\
\hline $\begin{array}{l}\text { DE Moniteur } \\
\text { Educateur }\end{array}$ & 465 & $92 \%$ & 166 & $57 \%$ & $26 \%$ & $17 \%$ & $19 \%$ & $44 \%$ \\
\hline $\begin{array}{l}\text { Bac Pro } \\
\text { Secrétariat }\end{array}$ & 429 & $97 \%$ & 255 & $73 \%$ & $23 \%$ & $4 \%$ & $29 \%$ & $55 \%$ \\
\hline BP Coiffure & 408 & $99 \%$ & 245 & $36 \%$ & $55 \%$ & $9 \%$ & $19 \%$ & $50 \%$ \\
\hline $\begin{array}{l}\text { BTS Assistant } \\
\text { de Gestion } \\
\text { PME-PMI }\end{array}$ & 386 & $90 \%$ & 225 & $74 \%$ & $15 \%$ & $11 \%$ & $30 \%$ & $58 \%$ \\
\hline $\begin{array}{l}\text { DE Aide } \\
\text { Medico- } \\
\text { Psychologique }\end{array}$ & 382 & $87 \%$ & 24 & $32 \%$ & $57 \%$ & $11 \%$ & $15 \%$ & $\mathrm{Nd}$ \\
\hline $\begin{array}{l}\text { BTS Aide de } \\
\text { Direction }\end{array}$ & 312 & $94 \%$ & 228 & $62 \%$ & $24 \%$ & $14 \%$ & $17 \%$ & $49 \%$ \\
\hline $\begin{array}{l}\text { BTS } \\
\text { Comptabilité } \\
\text { Gestion des } \\
\text { organisations }\end{array}$ & 294 & $100 \%$ & 179 & $66 \%$ & $14 \%$ & $20 \%$ & $19 \%$ & $60 \%$ \\
\hline $\begin{array}{l}\text { Titre Assistant } \\
\text { de vie aux } \\
\text { Familles }\end{array}$ & 282 & $99 \%$ & 214 & $87 \%$ & $6 \%$ & $7 \%$ & $8 \%$ & $73 \%$ \\
\hline $\begin{array}{l}\text { BP JEPS Loisirs } \\
\text { tous Publics }\end{array}$ & 258 & $81 \%$ & 54 & $72 \%$ & $17 \%$ & $11 \%$ & $16 \%$ & $45 \%$ \\
\hline
\end{tabular}

Champ : Candidats à la VAE résidant en Région Rhône-Alpes qui ont établi un dossier et obtenu une décision de

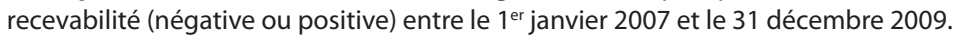

nd: non disponible.

Lecture : $87 \%$ des candidats passés en jury pour le titre d'Assistant de vie aux familles ont une validation totale, et parmi les candidats recevables, $73 \%$ ont bénéficié d'un accompagnement.

Sigles : CAP : Certificat d'Aptitude Professionnelle, DE : Diplôme d'Etat, BTS : Brevet de Technicien Supérieur, BPJEPS : Brevet Professionnel de la Jeunesse, de l'Education Populaire et du Sport.

Source : Base Résovalideur de la région Rhône-Alpes. 
A titre de comparaison, pour ces BTS, ce taux de réussite est supérieur à celui des candidats issus de la formation continue ${ }^{4}$. En revanche, pour le CAP Petite enfance et le bac pro Secrétariat, les taux d'obtention du diplôme par la VAE sont beaucoup plus faibles que via la formation continue (respectivement $94 \%$ et $88 \%$ ). Les certifications des secteurs médico-sociaux présentent des taux de validation totale relativement faibles au profit de validations partielles. Par exemple, le DE d'aide soignant, le diplôme le plus demandé, n’a un taux de validation totale que de $32 \%$ contre $57 \%$ pour la validation partielle. C'est aussi le cas du DE d'auxiliaire de puériculture ou du DE d'aide médico-psychologique. De même, le BP Coiffure apparaît particulièrement sélectif en VAE, puisque son taux de réussite est de $36 \%$ alors qu'il est de $68 \%$ en formation continue 5 . Plus globalement, nous observons que la proportion de candidats visant une certification de niveau $\mathrm{V}$ diminue au fur et à mesure de l'avancée dans le processus : il y a une baisse de plus de $6 \%$ pour ce niveau de diplôme entre l'entrée dans le dispositif et la décision de validation. Parmi les candidats visant un diplôme de niveau $\mathrm{V}$, une forte proportion n'avait aucun diplôme et donc a pu fortement sous-estimer les exigences et le travail réflexif nécessaires à la rédaction des dossiers de VAE.

Au vu de certains taux de validation, le dispositif VAE semble avoir induit des modifications dans l'arbitrage entre les différents modes d'acquisition des certifications.

Par exemple, en Rhône-Alpes, pour le diplôme d'auxiliaire de vie sociale, depuis 2007, la VAE a dépassé la formation initiale, en termes de diplômes délivrés (Grenat, 2010 ; Grenat et Nahon 2011). La VAE représente environ $25 \%$ des diplômes d'Educateur spécialisé délivrés, $13 \%$ du CAP Petite enfance et $8 \%$ des BP Coiffure (Abriac, 2011).

\section{Entretien conseil et accompagnement, deux atouts dans la réussite d'une VAE ?}

Au-delà des caractéristiques individuelles des candidats (voir Havet, 2014), nous pouvons nous interroger sur l'influence de caractéristiques propres à la démarche de VAE, sur lesquelles les pouvoirs publics pourraient jouer pour dynamiser le dispositif.

En particulier, il serait intéressant de savoir si les candidats ayant bénéficié d'un entretien conseil au sein d'un Point Relais Information Conseil (PRIC) ou d'un accompagnement réussissent mieux aux différentes étapes de VAE. Malheureusement, le rôle de l'accompa-

\footnotetext{
4. Les taux de réussite en formation continue sont respectivement de $55 \%$ pour le BTS Comptabilité des organisations et de $52 \%$ pour le BTS Assistant de gestion PME-PMI (Calculs à partir de la base Reflet du Céreq pour 2011).

5. Calcul à partir de la base Reflet du Céreq pour 2011-2012.

6. Havet (2014) met en évidence les différences de parcours VAE en fonction de l'âge, du sexe, du diplôme et du statut professionnel du candidat.
} 
gnement ne pourra être analysé que pour seulement trois ministères (Education nationale, Jeunesse et Sports, Emploi) (encadré 1) pour des raisons de disponibilité des données.

Il a été souvent avancé que les entretiens conseils au sein des PRIC pourraient être déterminants dans le processus de VAE; ils permettraient de mieux orienter les candidats parmi l'ensemble des certifications proposées au regard de leurs expériences professionnelles passées (Besson, 2008). Néanmoins, leur influence réelle sur les chances de réussite aux étapes de recevabilité et de validation a été peu analysée statistiquement, faute de données individuelles permettant d'observer un parcours VAE sur toute sa durée. En outre, parmi les difficultés inhérentes au dispositif, la durée du parcours est fréquemment soulignée. En moyenne nationale, environ deux années s'écoulent entre les premières informations et le passage devant le jury. Cette temporalité est souvent identifiée comme un facteur de démoralisation et donc comme un obstacle aux réussites (Beaupère et Podevin, 2012).

Dans ce contexte, les prestations d'accompagnement semblent pouvoir faciliter l'obtention du diplôme en réduisant la complexité du travail attendu dans le dossier de preuve et surtout la durée du parcours de VAE ; en effet, les rendez-vous successifs avec les accompagnateurs permettent de mieux mobiliser les candidats dans le travail de rédaction attendu (Labruyère et Quintero, 2009). Bonaïti (2008) et Labruyère (2009) ont étudié cette question mais uniquement sur des candidats visant des certifications de niveau V. Selon eux, l'accompagnement augmentait significativement les chances de validation totale.

\subsection{L'accompagnement, un meilleur atout de réussite ?}

Pour nos trois valideurs étudiés, $20 \%$ des candidats ayant déposé un dossier ont bénéficié d'un entretien conseil au sein d'un PRIC. Parmi les candidats ayant un dossier jugé recevable, $8 \%$ ont juste bénéficié d'un entretien conseil au sein d'un PRIC, $36,5 \%$ ont seulement reçu un accompagnement et $12,5 \%$ ont bénéficié des deux types de mesure. Ainsi, près d'un candidat sur deux recevable (49\%) a bénéficié de prestations d'accompagnement. Toutefois, le tableau 2 montre que «les candidats sont inégalement soutenus dans leur démarche de VAE», comme le titraient déjà Labruyère et Quintero (2009). Par exemple, les demandeurs d'emploi ont, en proportion, beaucoup plus souvent bénéficié d'entretiens conseils au sein des PRIC (+ 20 points) et de prestations d'accompagnement (+ 8 points) que les salariés. Il ressort aussi que les chances d'être accompagné augmentent fortement avec l'âge et diminuent avec le niveau de qualification du diplôme visé.

Plus précisément, parmi les 15 certifications les plus demandées, le titre d'Assistant de vie aux familles, de niveau $\mathrm{V}$ et avec le meilleur taux de validation totale, est le diplôme ayant le taux d'accompagnement le plus élevé (73\%). En revanche, les candidats à 
ce titre ont peu bénéficié d'un entretien conseil au sein d'un PRIC ; sans doute que beaucoup d'entre eux préparent cette certification dans le cadre d'un projet collectif de VAE. Les trois diplômes dont les taux d'accompagnement sont les plus élevés (variant entre $55 \%$ et $60 \%$ ) ne sont pas de niveau V : il s'agit du BTS Comptabilité gestion des organisations, du BTS Assistant de gestion PME-PMI et du bac pro Secrétariat, qui font partie des diplômes ayant les taux de validation totale les plus forts.

Tableau 2 : Taux d'entretiens conseils au sein d'un PRIC et d'accompagnement

\begin{tabular}{|c|c|c|}
\hline & $\begin{array}{l}\text { Entretiens conseils } \\
\text { (en \%) }\end{array}$ & $\begin{array}{c}\text { Accompagnement } \\
\left(\text { en } \%{ }^{\circ}\right)\end{array}$ \\
\hline Ensemble & 20 & 49 \\
\hline \multicolumn{3}{|c|}{ Statut de la personne } \\
\hline Demandeur d'emploi & 36 & 55 \\
\hline Salarié & 15 & 48 \\
\hline $\begin{array}{l}\text { Non salarié (bénévole, } \\
\text { retraité, inactivité) }\end{array}$ & 20 & 38 \\
\hline \multicolumn{3}{|l|}{ Age } \\
\hline$<30$ ans & 18 & 42 \\
\hline $30-39$ ans & 20 & 47 \\
\hline $40-44$ ans & 21 & 51 \\
\hline$>45$ ans & 22 & 52 \\
\hline \multicolumn{3}{|c|}{ Niveau du diplôme visé } \\
\hline Niveau I ou II & 13 & 13 \\
\hline Niveau III & 20 & 48 \\
\hline Niveau IV & 22 & 47 \\
\hline Niveau V & 19 & 53 \\
\hline Nombre d'observations & 10930 & 10126 \\
\hline
\end{tabular}

Champ : 1ère colonne : Candidats à la VAE résidant en Région Rhône-Alpes qui ont établi un dossier et obtenu une décision de recevabilité (négative ou positive) entre le 1er janvier 2007 et le 31 décembre 2009, pour les secteurs de l'Enseignement Secondaire, Jeunesse et Sport ou ministère de l'Emploi ; 2ème colonne : parmi ces candidats, ceux qui ont obtenu une recevabilité positive.

Lecture : 36 \% des demandeurs d'emploi ont bénéficié d'un entretien conseil au sein d'un PRIC et 55 \% des chômeurs recevables ont reçu un accompagnement.

PRIC : Point relais d'information conseil.

Source : Base Résovalideur de la région Rhône-Alpes. 
Le tableau 3 met en évidence l'influence des entretiens conseils et de l'accompagnement sur les taux de recevabilité positive, sur les chances d'être passé devant le jury et d'avoir obtenu une validation totale. Ainsi, les candidats qui ont bénéficié d'un entretien conseil au sein d'un PRIC ont des taux de recevabilité positive similaires à ceux n'en ayant pas bénéficié. De même, la différence dans les taux de passage en jury est faible entre ces deux groupes. Toutefois, elle masque de fortes disparités qui apparaissent quand les prestations d'accompagnement sont considérées simultanément (tableau 3). Certes, les candidats n'ayant bénéficié ni d'un entretien conseil ni d'une prestation d'accompagnement ont un taux moyen de passage en jury beaucoup plus faible que ceux ayant bénéficié de ces deux mesures d'aide ; mais de manière plus surprenante, ils ont un taux moyen de passage en jury plus élevé que les candidats ayant seulement bénéficié d'un entretien conseil.

Cette situation pourrait en partie refléter un phénomène de sélection : ce sont probablement les candidats qui ont le plus de doutes sur leur dossier et leur expérience (par exemple du fait de parcours professionnels chaotiques) qui ont demandé à être conseillés par un PRIC et donc pour qui la rédaction du livret est a priori plus compliquée. En particulier, la part des chômeurs est de 17 \% parmi ceux qui n'ont eu ni entretien conseil ni accompagnement, alors qu'elle s'élève à $39 \%$ parmi ceux ayant bénéficié des deux mesures et à $48 \%$ parmi ceux qui ont seulement bénéficié d'un entretien conseil.

L'accompagnement semble la mesure la plus décisive pour réduire le délai entre l'étape de recevabilité et le passage en jury; en effet, ceux qui en ont bénéficié ont des taux de passage d'environ $70 \%$, soit entre 25 et 35 points de plus par rapport à ceux n'en ayant pas reçu.

Pour la validation totale, les candidats ayant bénéficié d'un entretien conseil présentent des taux de réussite statistiquement inférieurs, sans doute là encore en partie à cause de certaines de leurs caractéristiques individuelles défavorables, notamment leur situation vis-à-vis de l'emploi. Les prestations d'accompagnement semblent un meilleur atout de réussite. Néanmoins, ces résultats "bruts " ne permettent pas de conclure définitivement que les prestations d'accompagnement facilitent davantage les chances de réussite d'une VAE que les entretiens conseil. Pour raisonner « toutes choses égales par ailleurs ", il convient de contrôler les effets conjoints des variables individuelles, telles que le statut des candidats vis-à-vis de l'emploi, le niveau de diplôme visé, l'âge, etc. 


\section{Tableau 3 : Taux de réussite aux différentes étapes selon que l'on ait bénéficié d'un entretien conseil au sein d'un Point Relais Information Conseil (PRIC) et/ou d'un accompagnement}

\begin{tabular}{lcccc}
\hline & $\begin{array}{c}\text { Recevabilité } \\
\text { positive }\end{array}$ & Passage en jury & $\begin{array}{c}\text { Validation } \\
\text { partielle }\end{array}$ & $\begin{array}{c}\text { Validation } \\
\text { totale }\end{array}$ \\
\hline $\begin{array}{l}\text { A reçu un entretien conseil au sein d'un PRIC } \\
\text { Non }\end{array}$ & $93 \%$ & $56 \%$ & $21 \%$ & $69 \%$ \\
Oui & $93 \%$ & $55 \%$ & $25 \%$ & $63 \%$ \\
& & & & \\
A reçu & -- & & & \\
Ni entretien conseil ni accompagnement & -- & $44 \%$ & $22 \%$ & $66 \%$ \\
Juste un entretien conseil au sein d'un PRIC & -- & $71 \%$ & $25 \%$ & $61 \%$ \\
Seulement des prestations & --- & $69 \%$ & $50 \%$ & $64 \%$ \\
d'accompagnement & & & & \\
Un entretien conseil et un accompagnement & & & $50 \%$ \\
\hline
\end{tabular}

Champ : Candidats ayant obtenu une décision de recevabilité sur leur dossier VAE en 2007, 2008 ou 2009 en région Rhône-Alpes pour les trois secteurs suivants : Education nationale du secondaire (DAVA Grenoble et DAVA Lyon), Jeunesse et Sport (DRJS Lyon) et Travail et Emploi (ministère de l'Emploi). Lecture : Parmi les candidats à la VAE ayant bénéficié d'un entretien conseil au sein d'un PRIC (Point relai d'information conseil), $93 \%$ ont obtenu une recevabilité positive à leur dossier. Pour les candidats ayant été jugés recevables, le taux de passage en jury est de $69 \%$ pour ceux qui ont bénéficié à la fois d'un entretien conseil et d'un accompagnement, contre seulement $44 \%$ pour ceux n'ayant bénéficié d'aucune de ces mesures.

Source : Base Résovalideur de la région Rhône-Alpes.

\subsection{Etre salarié, un atout pour la réussite}

Les résultats des modèles Logit ${ }^{7}$ du tableau 4 confirment les études précédentes (Bonaïti, 2008 ; Havet, 2014) sur le rôle de certaines caractéristiques individuelles dans les parcours de VAE. Par exemple, un vrai mécanisme de différenciation semble être à l'œuvre à toutes les étapes du dispositif de VAE, en fonction du statut professionnel. Par rapport aux demandeurs d'emploi, les salariés ont des probabilités plus élevées d'obtenir une recevabilité positive de leur dossier (+ 4 points), de passer en jury (+ 12 points) et d'obtenir une validation totale de la certification visée (+ 8 points), toutes choses égales par ailleurs. En effet, les VAE à l'initiative de l'employeur, comme les "VAE collectives ", proposent souvent, comme nous l'avons vu, une logistique mobilisée pour l'ensemble des candidats, même si la rédaction du dossier reste individuelle; cela permet d'optimiser les délais et les chances de réussite (Beaupère et Podevin, 2012). De même, pour les salariés, la VAE

7. Le Logit est un modèle de régression binomiale qui permet de modéliser adéquatement les réponses binaires. Il permet de quantifier l'impact propre de chaque facteur sur la variable à expliquer (ici respectivement : le fait d'avoir été recevable, le fait d'être passé en jury et le fait d'avoir obtenu une validation totale). 
peut s'intégrer à la mise en place de démarches de gestion prévisionnelle des emplois et des compétences (GPEC), les impliquant dans la construction de référentiels. Le travail d'explication et de mise en visibilité des compétences réalisé à cette occasion correspond à celui qui est demandé aux candidats à la VAE lors de l'élaboration de leur dossier. Ainsi les salariés associés dans leur entreprise à une démarche GPEC seraient mieux préparés pour la VAE (Beaupère et Podevin, 2008).

En outre, comme le souligne Bonaïti (2008), l'expérience des demandeurs d'emploi étant plus lointaine que celle des salariés, il leur est sans doute plus difficile de décrire avec précision leurs compétences. Ces dernières peuvent aussi, pour certains, se révéler obsolètes par rapport aux certifications visées. Les salariés ont enfin probablement plus facilement accès, dans leur entreprise, à des documents leur permettant de renseigner leur dossier (Beaupère et Podevin, 2012). Pour toutes ces raisons, il n'est pas surprenant que le fait d'être salarié représente un réel atout dans les chances de réussite aux différentes étapes du processus de VAE.

A autres caractéristiques équivalentes, les hommes ont plus de chances d'obtenir une recevabilité positive à leur dossier que les femmes, mais ils ont des probabilités plus faibles de se présenter en jury sur la période d'observation, une fois l'étape de recevabilité passée. Toutefois, parmi les candidats qui se sont présentés en jury, les hommes bénéficient de décisions de validation de leur acquis d'expérience plus favorables que les femmes.

Quant à l'effet de l'âge et du niveau de diplôme, le tableau 4 révèle que les individus les plus âgés qui visent une certification de niveau $V$ ou de niveau III et plus ont davantage de chances d'être passés en jury. Pour la validation totale, les individus de plus de 40 ans qui visent un diplôme de niveau bac ou plus ont les meilleurs taux de réussite, toutes choses égales par ailleurs.

De plus, nos résultats révèlent que les taux de réussite aux différentes étapes du parcours de VAE sont significativement plus élevés pour les diplômes du ministère de l'Emploi, que pour ceux de l'Enseignement secondaire, eux-mêmes étant plus élevés que ceux des diplômes du secteur Jeunesse et Sport.

Les résultats du tableau 4 témoignent surtout qu'une fois l'effet des caractéristiques individuelles contrôlées, les prestations d'accompagnement constituent de réels atouts dans la démarche de VAE d'un candidat au niveau des étapes les plus exigeantes (la rédaction du livret et l'entretien). A profil de candidat équivalent, sur la période d'observation, les candidats ayant bénéficié d'un accompagnement ont une probabilité d'être passés en jury beaucoup plus élevée que ceux n'en ayant pas bénéficié $(+27$ points). Ainsi, les prestations d'accompagnement accélèreraient la procédure de VAE, une fois celle-ci entamée. L'accompagnement semble crucial pour une rédaction rapide du dossier de VAE et une réduction du délai de la procédure. La durée entre les déci- 
sions de recevabilité et le passage en jury serait en moyenne divisée par deux lorsque le candidat bénéficie de telles prestations. En outre, il tend à accroître la probabilité d'obtenir une validation totale ( +4 points). Nos résultats sont cohérents avec ceux de l'étude de Bonaïti (2008) pour les diplômes de niveau V.

Les modèles Logit, qui permettent une analyse en tenant compte de certaines particularités de la population conseillée au sein d'un PRIC, relativisent l'impact des entretiens conseils, observé dans le tableau 3. Lorsque nous raisonnons à situation vis-à-vis de l'emploi, du sexe, de l'âge, de niveau de diplôme visé équivalents, il ressort que les candidats ayant bénéficié d'un entretien conseil au sein d'un PRIC ont un taux moyen de validation totale similaire à ceux n'en ayant pas bénéficié ; cependant, ils ont une probabilité plus faible d'être passés en jury (- 6 points).

Ce dernier résultat mériterait d'être étayé pour en déterminer l'origine grâce à des informations plus précises sur l'expérience du candidat, voire grâce à des données qualitatives. En effet, plusieurs hypothèses pouvant être formulées restent à tester.

D'une part, nous pouvons supposer que les candidats sollicitant un entretien conseil au sein d'un PRIC ont eu des parcours professionnels plus morcelés ou chaotiques; ils mettraient donc naturellement plus de temps à décrire toutes les compétences acquises et à rédiger leur livret de VAE ou pourraient être plus enclins à abandonner avant l'étape du jury. Par ailleurs, il y a des chances qu'ils soient moins engagés dans des VAE collectives ou VAE initiées par les entreprises, qui favorisent les chances de réussite (Beaupère et Podevin, 2012). Par conséquent, l'entretien conseil ajouterait une étape qui alourdirait le parcours et pourrait ainsi décourager les candidats les plus fragiles.

D'autre part, il est possible que l'entretien conseil ait engendré un meilleur travail réflexif des candidats sur leurs activités, susceptible de leur avoir permis d'atteindre leur objectif (changement d'emploi, retour à l'emploi pour les chômeurs, etc), sans qu’ils aient nécessairement mené le processus jusqu’à la certification. Des données détaillées sur les parcours professionnels des candidats et sur les phénomènes et raisons d'abandon semblent indispensables pour mieux comprendre le rôle des entretiens conseils dans le dispositif de VAE. 
Tableau 4 : Probabilités d'obtenir une recevabilité positive, d'être passé en jury et d'obtenir une validation

\begin{tabular}{|c|c|c|c|}
\hline & \multicolumn{3}{|c|}{ Variation de probabilité entraînée (en points) pour } \\
\hline & $\begin{array}{c}\text { Recevabilité } \\
\text { positive }\end{array}$ & Passage en jury & Validation totale \\
\hline $\begin{array}{l}\text { Individu de référence (probabilité estimée) } \\
\text { Sexe Femmes } \\
\text { Hommes }\end{array}$ & $\begin{array}{l}88 \% \\
\text { réf. } \\
+1,8\end{array}$ & $\begin{array}{l}30 \% \\
\text { réf. } \\
-5,8\end{array}$ & $\begin{array}{l}66 \% \\
\text { réf. } \\
+7,4\end{array}$ \\
\hline $\begin{array}{l}\text { Niveau de diplôme visé et âge } \\
\text { Niveau V et }<40 \text { ans } \\
\text { Niveau V et } 40-44 \text { ans } \\
\text { Niveau V et }>45 \text { ans } \\
\text { Niveau IV et }<40 \text { ans } \\
\text { Niveau IV et }>40 \text { ans } \\
\text { Niveau I, II ou III et }<40 \text { ans } \\
\text { Niveau I, II ou III et } 40-44 \text { ans } \\
\text { Niveau I, II ou III et }>45 \text { ans }\end{array}$ & $\begin{array}{c}\text { réf. } \\
+9,1 \\
+9,5 \\
+9,8 \\
+4,0 \\
+10,8 \\
-5,4 \\
\text { ns }\end{array}$ & $\begin{array}{c}\text { réf. } \\
+4,3 \\
+4,2 \\
\text { ns } \\
\text { ns } \\
\text { ns } \\
\text { ns } \\
+4,9\end{array}$ & $\begin{array}{c}\text { réf. } \\
\text { ns } \\
\text { ns } \\
+5,3 \\
+11,3 \\
\text { ns } \\
+5,4 \\
+6,5\end{array}$ \\
\hline $\begin{array}{l}\text { Statut de la personne } \\
\text { Demandeur d'emploi } \\
\text { Salarié } \\
\text { Non salarié (bénévole, retraité, inactivité) }\end{array}$ & $\begin{array}{l}\text { réf. } \\
+3,5 \\
+4,0\end{array}$ & $\begin{array}{l}\text { réf. } \\
+11,9 \\
+12,4\end{array}$ & $\begin{array}{l}\text { réf. } \\
+79 \\
\text { ns }\end{array}$ \\
\hline $\begin{array}{l}\text { Secteur du valideur } \\
\text { Education nationale, Enseignement secondaire } \\
\text { Jeunesse et Sports } \\
\text { Travail - Emploi }\end{array}$ & $\begin{array}{l}\text { réf. } \\
-31,4 \\
+6,6\end{array}$ & $\begin{array}{l}\text { réf. } \\
-15,5 \\
+5,3\end{array}$ & $\begin{array}{l}\text { réf. } \\
-18,2 \\
+17,8\end{array}$ \\
\hline Nombre de dossiers déposés par diplôme & & ns & \\
\hline Durée depuis la date de recevabilité & & $+0,3$ & \\
\hline $\begin{array}{l}\text { A reçu entretien conseil au sein d'un PRIC } \\
\text { Oui } \\
\text { Non }\end{array}$ & $\begin{array}{l}\text { ns } \\
\text { réf. }\end{array}$ & --- & --- \\
\hline $\begin{array}{l}\text { A reçu } \\
\text { Ni entretien conseil ni accompagnement } \\
\text { Juste un entretien conseil au sein d'un PRIC } \\
\text { Juste des prestations d'accompagnement } \\
\text { Un entretien conseil et des prestations d'accompagnement }\end{array}$ & $\begin{array}{l}--- \\
--- \\
--- \\
---\end{array}$ & $\begin{array}{l}\text { réf. } \\
-6,1 \\
+27,0 \\
+27,0\end{array}$ & $\begin{array}{l}\text { réf. } \\
\text { ns } \\
+4,1 \\
\text { ns }\end{array}$ \\
\hline
\end{tabular}




\begin{tabular}{lccc}
\hline & $\begin{array}{c}\text { Recevabilité } \\
\text { positive }\end{array}$ & Passage en jury & Validation totale \\
\hline Nombre d'observations & 10795 & 10003 & 5613 \\
\hline
\end{tabular}

Note : Le recours à une modélisation de type Logit permet d'isoler l'effet spécifique d'un facteur « toutes choses étant égales par ailleurs ». La significativité est établie au seuil de $5 \%$; ns : non significatif.

La situation de référence est celle d'une demandeuse d'emploi, ayant obtenu une décision de recevabilité en 2009 dans le département de l'Ain, âgée entre 40 et 44 ans, visant un diplôme de niveau V de l'Education nationale et n'ayant bénéficié ni d'un entretien conseil du PRIC (Point relais d'information conseil), ni d'un accompagnement. Par souci de concision dans la présentation, les effets des départements et des années n'ont pas été reportés mais ces variables ont bien été intégrées dans la régression.

Lecture : Pour l'individu de référence, la probabilité d'avoir une recevabilité positive est de $88 \%$. Pour une femme salariée ayant les mêmes caractéristiques, cette probabilité est supérieure de 3,5 points.

Champ : $1^{\text {ère }}$ colonne : candidats ayant reçu une décision de recevabilité ,en 2007-2009, en région Rhône-Alpes, aux diplômes de l'Education nationale du secondaire, de Jeunesse et Sport et du ministère de l'Emploi ; $2^{\text {ème }}$ colonne : restriction à seulement ceux qui ont eu une recevabilité positive ; $3^{\text {ème }}$ colonne : restriction à ceux qui sont effectivement passés en jury.

Sigles : Niveau I ou II : diplôme supérieur à bac + 2 (licence, maîtrise, master, DEA, DESS - respectivement diplôme d'études approfondies, diplôme d'études supérieures spécialisées --, doctorat); Niveau III : niveau bac + 2 (DUT, BTS, DEUG - respectivement diplôme universitaire de technologie, brevet de technicien supérieur, diplôme d'études universitaires générales) ; niveau IV : niveau bac, niveau V : CAP, BEP (respectivement certificat d'aptitude professionnelle, brevet d'études professionnelles) ou équivalent.

Source : Base Résovalideur de la région Rhône-Alpes.

\section{Conclusion}

Ainsi, l'exigence de certification se généralisant à tous les métiers, la Validation des Acquis de l'Expérience (VAE), telle qu'instituée par la loi de Modernisation Sociale du 17 janvier 2002, devait constituer une réelle opportunité pour les adultes n'ayant pas eu la possibilité d'obtenir un diplôme en formation initiale ou n'ayant pas le temps nécessaire pour suivre un cursus complet de formation continue.

La VAE met en effet l'expérience professionnelle sur un même pied d'égalité avec la formation initiale, la formation continue et la formation en alternance. L'objectif affiché de la VAE par le législateur était double : d'une part, les actifs concernés, et notamment ceux aux parcours professionnels les plus chaotiques, pourraient espérer une amélioration de leurs perspectives de carrières ; d'autre part, les entreprises parviendraient à mieux identifier les compétences des candidats dans un emploi et être ainsi plus réactives et efficaces dans un monde professionnel aux changements technologiques et réglementaires permanents.

Notre étude a utilisé les données sur l'ensemble des individus ayant déposé un dossier de candidature à la VAE, entre 2007 et 2009, auprès des principaux valideurs de la région Rhône-Alpes. Il s'agissait de mettre en lumière la sélection s'opérant au fur et à mesure de l'avancée dans le dispositif. 
Avec près d'un candidat sur deux visant un diplôme de niveau V, la VAE apparaît relativement conforme à l'un des vœux des promoteurs de ce dispositif, qui souhaitaient favoriser les trajectoires professionnelles des adultes peu qualifiés.

En revanche, la VAE semble peu encline à jouer un véritable rôle d'insertion professionnelle pour les chômeurs : les demandeurs d'emploi représentent seulement $20 \%$ des candidats à la VAE mais, surtout, ils rencontrent plus de difficultés à réussir à chacune des étapes de la procédure. La longueur de la procédure, avec un délai entre l'étape de recevabilité et celle de présentation en jury oscillant le plus souvent entre un et deux ans, n'est pas toujours très adaptée au contexte de recherche d'emploi. Par exemple, elle pourrait entraver un retour rapide à l'emploi des chômeurs qui espéraient obtenir, par la VAE, une certification exigée pour postuler à des professions "réglementées ". Néanmoins, il est fort probable que le retour à l'emploi de certains chômeurs soit facilité par le dispositif de VAE, non pas par la certification en elle-même mais par le travail réflexif sur leurs activités, y compris quand ils ne mènent pas la procédure jusqu'au bout. Seules des enquêtes de suivi des trajectoires professionnelles des candidats à la VAE, avant l'entrée dans le dispositif et une fois sortis, permettraient de répondre à ces interrogations.

Dans ce contexte de la VAE, où les principaux facteurs d'abandon semblent être la durée et la complexité de la procédure, les prestations d'accompagnement ont un véritable rôle à jouer, qu'il faut sans doute renforcer et cibler sur les publics les plus en difficulté, notamment les demandeurs d'emploi.

En effet, l'accompagnement aurait des retombées positives sur les chances de réussite dans la démarche de VAE. Il réduirait fortement le délai entre la décision de recevabilité et le passage en jury. Il serait donc à rendre davantage systématique, et en particulier pour faire aboutir les demandes de validation des demandeurs d'emploi.

Nos résultats, même partiels et qui mériteraient d'être consolidés par des données plus globales, confortent l'idée que les prestations d'accompagnement sont primordiales. En effet, un candidat encadré et suivi aura plus de facilités à constituer et rédiger son dossier et augmentera ainsi ses chances de voir sa démarche aboutir rapidement à une validation totale. Toutefois, pour véritablement utiliser l'accompagnement comme moyen d'action pour améliorer l'efficacité de la VAE, il conviendrait de réaliser des études complémentaires se focalisant sur le contenu, la qualité et l'intensité des prestations reçues ${ }^{8}$. Elles permettraient de formuler des préconisations précises sur le type d'accompagnement à proposer pour différentes populations cibles. Nos résultats mettent aussi en évidence que les actions de conseil au sein des PRIC (Point relais information conseil), en amont de la validation des acquis proprement dite, s'avèrent non négligeables pour une éventuelle montée en puissance de ce dispositif. Plus l'information sur le processus de VAE sera étendue auprès des

8. Il est à noter que la DRJSC a déjà mis en place un questionnaire évaluatif auprès des candidats accompagnés afin de faire progresser la qualité de son accompagnement. 
candidats potentiels, plus le nombre de dépôts de dossiers est susceptible d'augmenter. Par ailleurs, ces entretiens conseils constitueraient une occasion de promouvoir, auprès des candidats, les prestations d'accompagnement, qui semblent être une clé de la réussite dans le dispositif de VAE.

\section{Bibliographie}

Abriac D. (2011), «Diplômes du second degré technologique et professionnel : la demande de VAE reste très concentrée ", DEPP Note d'information, 11.04, janvier.

Ballet V. (2009), "La validation des acquis de l'expérience (VAE) : entre gestion individuelle et organisationnelle des carrières ", Revue multidisciplinaire sur l'emploi, le syndicalisme et le travail, vol. 4, $\mathrm{n}^{\circ}$ 1, pp. 62-83.

Beaupère N. et Podevin G. (2012), " Pour un accompagnement global des parcours de VAE », Bref-Céreq, n 302.

Beque M. (2010), " La VAE en 2009 au ministère chargé de l'Emploi ", Dares-Analyses, $\mathrm{n}^{\circ} 83$, décembre.

Besson E. (2008), Valoriser l'acquis de l'expérience : une évaluation du dispositif de VAE, Rapport au premier ministre du secrétariat d'Etat chargé de la prospective, de l'évaluation des politiques publiques et du développement de l'économie numérique, septembre, $76 \mathrm{p}$.

Bonaïti C. (2008), « Le parcours des candidats à la validation par les acquis de l'expérience des titres et diplômes de niveau $\mathrm{V}$ : mieux vaut être accompagné et diplômé pour réussir ", Premières Synthèses, n 34.2, août, DARES.

De Rozario P. (2013), "Politique et management de la VAE dans le travail social ", Formation Emploi, n 122, Avril-Juin, pp. 87-114.

Giret J.-F. et Lemistre P. (2008), "Les théories du filtre et du signalement ", dans Paul J.-J. et Rose J., Le tour des relations formation-emploi-travail en cinquante-cinq questions, édition Dunod.

Grenat P. (2010), «La formation aux professions sociales en 2007 », Document de Travail DRESS, Série Statistiques, $\mathrm{n}^{\circ} 145$, juin.

Grenat P. et Nahon S. (2011), "La formation aux professions sociales en 2008 et 2009 ", Document de Travail DRESS, Série Statistiques, ${ }^{\circ}$ 54, mars.

Havet N. (2014), « Les bénéficiaires de la validation des acquis de l'expérience : l'exemple de la région Rhône-Alpes ", Revue Economique, à paraître.

Kogut-Kubiak F., Morin C., Personnaz E. et al. (2006), "Les logiques d'accès à la VAE et parcours de validation ", Relief, Céreq, n 12, 114 p. 
Labruyère C. (2006a), « La VAE, quels candidats pour quels diplômes », Bref-Céreq, nº 230.

Labruyère C. (2006b), "VAE : une nouvelle voie d'accès aux diplômes en cours de vie professionnelle » dans Données Sociales, chapitre 2.

Labruyère C., Paddeu J., Savoyant A., Tessier J. et Rivoire B. (2002), " La validation des acquis professionnels: Bilans des pratiques actuelles, enjeux pour les dispositifs futurs ", Bref-Céreq, n 185.

Labruyère C. et Quintero N. (2009), " Des candidats inégalement soutenus dans leur démarche de VAE ", Bref-Céreq, n² 263.

Legrand Z. (2012), "La VAE en 2011 dans les ministères certificateurs : le nombre de diplômes délivrés reste stable ", Dares Analyses, n 91, décembre.

Le Roux A. (2012), " La VAE dans les établissements d'enseignement supérieur en 2011 », Note d'Information, 12.31, MEN-DEPP, décembre.

Personnaz E., Quintero N. et Séchaud F. (2005), « Parcours de VAE, des itinéraires complexes, longs, à l'issue incertaine ", Bref-Céreq, n² 224, novembre.

PRAO (2011), «Analyse des parcours VAE en Rhône-Alpes entre 2007 et 2009 : Candidats à la VAE : Quels parcours pour quels résultats ", Rapport final, juin.

Spence M. (2002), "Signaling in Retrospect and the Informational Structure of Markets", American Economic Review, vol. 92, n 3, pp. 434-459. 